\title{
ESTUDO DOS NÍVEIS DE PRESSÃO SONORA CAUSADOS PELA RESSONÂNCIA MAGNÉTICA EM UM HOSPITAL PÚBLICO EM PRESIDENTE PRUDENTE - SP
}

\author{
Cássio Fabian Sarquis de Campos, Kátia Akiko Miazaka Sakemi \\ Universidade do Oeste Paulista - UNOESTE. Radiologia, Presidente Prudente - SP. E-mail: cassio@unoeste.br
}

\section{RESUMO}

O presente trabalho tem como objetivo aferir os níveis de ruídos encontrados no setor de Radiodiagnóstico de um Hospital público em Presidente Prudente durante a realização de exames de Ressônância Magnética verificando também se estão dentro dos limites aceitáveis estabelecidos por lei para prática profissional desta modalidade de radiodiagnóstico. A análise dos resultados foi realizada através de comparações diretas das aferições obtidas durante e sem a realização de exames visando evidenciar possíveis aumentos nos níveis de pressão sonora durante as práticas de exames. Para realização das aferições utilizou-se aparelho medidor de nível de pressão sonora, marca MINIPA, modelo MSL-1325, ponderado em A de $40 \mathrm{~dB}$ à $130 \mathrm{~dB}$, SLOW, com frequência analisada de $125 \mathrm{~Hz}$ à $8000 \mathrm{~Hz}$, respeitando as orientações da NR-15. Conclui-se que do ponto de vista da exposição aos ruídos, no ambientes de trabalho do setor de ressonância magnética de um Hospital público em Presidente Prudente, o nível de pressão sonora não ultrapassou os limites de $85 \mathrm{~dB}(\mathrm{~A})$ recomendados pela NR-15 e a NHO-01 da FUNDACENTRO.

Palavras-chave: ressonância magnética ruído acústico nível pressão sonora

\section{STUDY OF SOUND PRESSURE LEVELS CAUSED BY MAGNETIC RESONANCE ON A PUBLIC HOSPITAL ON PRESIDENTE PRUDENTE-SP}

\begin{abstract}
This study aims to assess the levels of noise found in the sector of Radiology public hospital on Presidente Prudente while performing MRI exams also checking if they are within acceptable limits established by law for the professional practice of this type of diagnostic radiology. The analysis was performed by direct comparison of the measurements obtained during and without conducting tests aimed evidence of possible increases in sound pressure levels during the practical examination. To perform the measurements was used for sound pressure level, MINIPA brand, model MSL-1325 meter, A-weighted $40 \mathrm{~dB}$ to $130 \mathrm{~dB}$, SLOW often analyzed from $125 \mathrm{~Hz}$ to 8000 $\mathrm{Hz}$ unit, respecting the guidelines NR -15 . We conclude that from the point of view of noise exposure in the workplace, the MRI sector of public hospital on Presidente Prudente, the sound pressure level does not exceed the limits of $85 \mathrm{~dB}(\mathrm{~A})$ recommended by NR-15 and NHO-01 FUNDACENTRO.
\end{abstract}

Keywords: magnetic resonance acoustic noise sound pressure level 


\section{INTRODUÇÃO}

A ressonância magnética é um exame de diagnóstico por imagem que não possui propriedades ionizantes, tornando assim segura sua aplicação do ponto de vista de ionização celular (TINOIS, 2005). Segundo Fanton (2007), "a ressonância magnética é um método de emissão - resposta, onde um material que está para análise recebe um certo estímulo magnético e em resposta um sinal será captado por uma antena no aparelho e enviado ao processamento de dados de um computador, transformando-o em uma imagem".

Conforme Bontrager e Lampignano (2010), a base da ressonância magnética compreende a interação de ondas de rádio e campos magnéticos estáticos com o núcleo do átomo, estes núcleos para serem usados adequadamente na ressonância magnética precisam ter um número ímpar de próton ou nêutron, caso contrário, não responderão aos estímulos esperados no exame para geração de imagens.

O corpo humano é composto por átomos que são minúsculas partículas onde o mais simples deles e o mais utilizado para produção de imagens é o núcleo do Hidrogênio (próton) fato explicado pela quantidade existente nos seres biológicos, cerca de $10^{10}$ átomos por centímetros cúbico (AMARO JÚNIOR; YAMASHITA, 2001).

Li, Lim e Lee (2007), dizem que a RM é uma das variedades tecnológicas mais significativas na área médica, auxiliando no diagnóstico de doenças e pesquisas biomédicas, no entanto, para o seu funcionamento a ressonância magnética produz ruídos que podem ser danosos à audição, além de dificultar o contato paciente, podendo causar consequências psicofisológicas, incluindo mudanças temporárias no limiar auditivo, ansiedade, estresse, irritação, fadiga mental, bem como a perda permanente da audição.

Segundo Ravicz, et al. (2008) este ruído é provocado pela passagem da corrente elétrica nas bobinas de gradiente, e se constitui como um incômodo, podendo alcançar níveis tão elevados quanto $100 \mathrm{~dB}$, pois durante a aquisição da imagem, a ressonância magnética utiliza 3 conjuntos de bobinas que originam gradientes de campos magnéticos, a corrente que passa através destas bobinas geram ruídos intermitentes que duram praticamente o tempo do exame (RAVICZ; MELCHER; KIANG, 2008).

Gradientes são alterações do campo magnético principal e são gerados por bobinas localizadas no corpo do magneto (WESTBROOK; KAUT, 2000, p.34).

O ser humano possui cinco sentidos fundamentais, dentre estes está a audição responsável pela percepção sonora, permitindo ao indivíduo a capacidade de ouvir. O ouvido também é um 
dos responsáveis pelo equilíbrio do corpo através do sistema vestibular, comumente conhecido como labirinto (RUI; STEFFANI, 2007).

As ondas sonoras são vibrações que se propagam pelo ar, quando captadas pelo pavilhão auricular são conduzidas até a membrana timpânica fazendo-a vibrar, estas vibrações passam para os ossículos do ouvido médio na sequência martelo, bigorna e estribo, fazendo com que o líquido na cóclea (situada no ouvido interno) se movimente, consequentemente as células ciliadas situadas dentro da cóclea captam este movimento e através de impulsos nervosos o envia para o encéfalo onde será interpretado; o encéfalo está diretamente ligado ao nervo auditivo (RUI; STEFFANI, 2007).

De acordo com as autoras Leão e Dias (2010), entende-se por ruído um tipo de som desagradável, que interfere até na percepção de outros sons. Dependendo da intensidade e da exposição em excesso ao ruído podem acarretar efeitos nocivos ao ser humano, ocasionando lesão da extensão das vias auditivas, desde a membrana timpânica até regiões do sistema nervoso central assim como sintomas extra-auditivos, a saber alterações do sono; transtornos de comunicação; neurológicos; comportamentais.

Holsbach, Conto e Godoy (2001), afirmam ainda que a exposição excessiva ao ruído pode colocar em risco a integridade física e psicológica do ser humano, além de provocar cefaléia, frequência cardíaca e pressão arterial alta, contração muscular, irritabilidade, insônia, fadiga, falta de atenção, inclusive perda da audição.

Além da perda auditiva, o zumbido também é uma queixa comum em profissionais que atuam em ambientes ruidosos com níveis acima de $85 \mathrm{~dB}$ e sua prevalência aumenta de acordo com a evolução do dano auditivo (LOPES et al., 2009).

A transmissão e propagação do som no ar em termos físicos são flutuações de pequenas escalas de pressão do ar em torno da pressão atmosférica, estas flutuações são definidas como pressão sonora e sua unidade é Newton por metro quadrado $\left(\mathrm{N} / \mathrm{m}^{2}\right)$ ou Pascal $(\mathrm{Pa})$, quando avaliadas as intensidades destas flutuações ou energia obtém-se o nível de pressão sonora.

O nível da pressão sonora em uma escala linear é pouco prática, pois há uma grande disparidade na utilização dos números, para reduzir a discrepância destas grandezas acústicas foi estabelecida como medida relativa de intensidade onde a unidade de medida é o Bel (PORTELA, 2008).

Legislações acidentárias relacionadas ao trabalho foram registradas desde 1919, e somente através da promulgação do Decreto - Lei 5.452 de 1ำ de Março de 1943 da Consolidação das Leis 
do Trabalho (CLT) firmaram-se as várias normalizações do trabalho, com o propósito de prevenir doenças ocupacionais e acidentes de trabalho (PORTELA, 2008).

No Brasil, o artigo 180 da Consolidação das Leis do Trabalho, assegurou ao trabalhador o direito de evitar a fadiga auditiva, com o passar do tempo houve várias alterações para benefícios em relação ao conforto e segurança no ambiente de trabalho, entre estas está a Portaria no 3214, de Junho de 1978, que aprovou as Normas Regulamentadoras (NRs), responsáveis pelas regras de segurança nas atividades relativas ao trabalho em nível nacional (OLIVEIRA FILHO, 2011).

Higienistas do trabalho classificam ruído como um agente físico indesejável e seus limites de tolerância são estabelecidos pela NR-15, esta norma atesta que o trabalhador não sofrerá danos no exercício de sua função, se a exposição aos níveis de ruído estiver dentro dos limites estabelecidos (RODRIGUES, 2009).

A NBR-10151 estabelece os níveis aceitáveis de ruído em comunidades e determina método para sua medição. Para avaliação, emprega-se as medições do nível de pressão sonora equivalente (LAeq), em decibels ponderados em " $A$ ", denominado $\mathrm{dB}(A)$.

A NHO-01 Trata-se de um reestudo da equipe técnica da Coordenação de Higiene do Trabalho da FUNDACENTRO - Fundação Jorge Duprat Figueiredo de Segurança e Medicina do Trabalho, devido ao grande avanço tecnológico e surgimento de novas técnicas para identificação e controle dos riscos ambientais. Esta norma determina parâmetros para avaliação e controle da exposição ocupacional ao ruído para profissionais técnicos que trabalham na área da saúde ocupacional

Na Portaria no 453 do Ministério da Saúde de 1988, que baixa diretrizes para as práticas radiológicas no país quanto à proteção de seus indivíduos, observa-se a ausência de orientações quanto à proteção do trabalhador expostos a fontes de ruídos dentro do setor de diagnóstico por imagem.

O presente trabalho tem como objetivo aferir os níveis de ruídos encontrados no setor de Radiodiagnóstico de um Hospital público em Presidente Prudente durante a realização de exames de Ressônância Magnética verificando também se estão dentro dos limites aceitáveis estabelecidos por lei para prática profissional desta modalidade de radiodiagnóstico.

\section{METODOLOGIA}

O presente trabalho tem como tema o estudo de ruídos produzidos pelo aparelho de Ressonância Magnética durante a jornada de trabalho diária do profissional ocupacionalmente 
exposto que pode ser de 8 horas para enfermeiros e de 4 horas para técnicos ou tecnólogos em radiologia.

Para tanto, um estudo do local proporcionou a elaboração de um croqui e escolha dos locais de medidas. As aferições dos níveis de pressão sonora ocorreram em dias de trabalhos normais do setor de radiodiagnóstico e seguiram os estabelecidos pelas NBR-10152, nível de ruído para conforto acústico, a NBR-10151 como complemento, a NR-15, atividade e operações Insalubres e a NHO-01, Normas de Higiene Ocupacional.

A análise dos resultados foi realizada através de comparações diretas das aferições obtidas durante e sem a realização de exames visando evidenciar possíveis aumentos nos níveis de pressão sonora durante as práticas de exames de RM. Na análise, realizou-se ainda um estudo se os limites obtidos são aceitáveis e estabelecidos por lei.

Para realização das aferições utilizou-se aparelho medidor de nível de pressão sonora, marca MINIPA, modelo MSL-1325, ponderado em A de $40 \mathrm{~dB}$ à $130 \mathrm{~dB}$, SLOW, com frequência analisada de $125 \mathrm{~Hz}$ a $8000 \mathrm{~Hz}$, respeitando as orientações a NR-15.

A coleta de dados foi feita em dois momentos distintos, durante a realização de exame e sem a realização de exame nos locais assinalados no croqui seguinte em três pontos diferentes para cada ambiente adjacente à sala do aparelho.

Figura 1. Croqui da classificação das áreas do Setor de Radiodiagnóstico de um Hospital público em Presidente Prudente-SP.

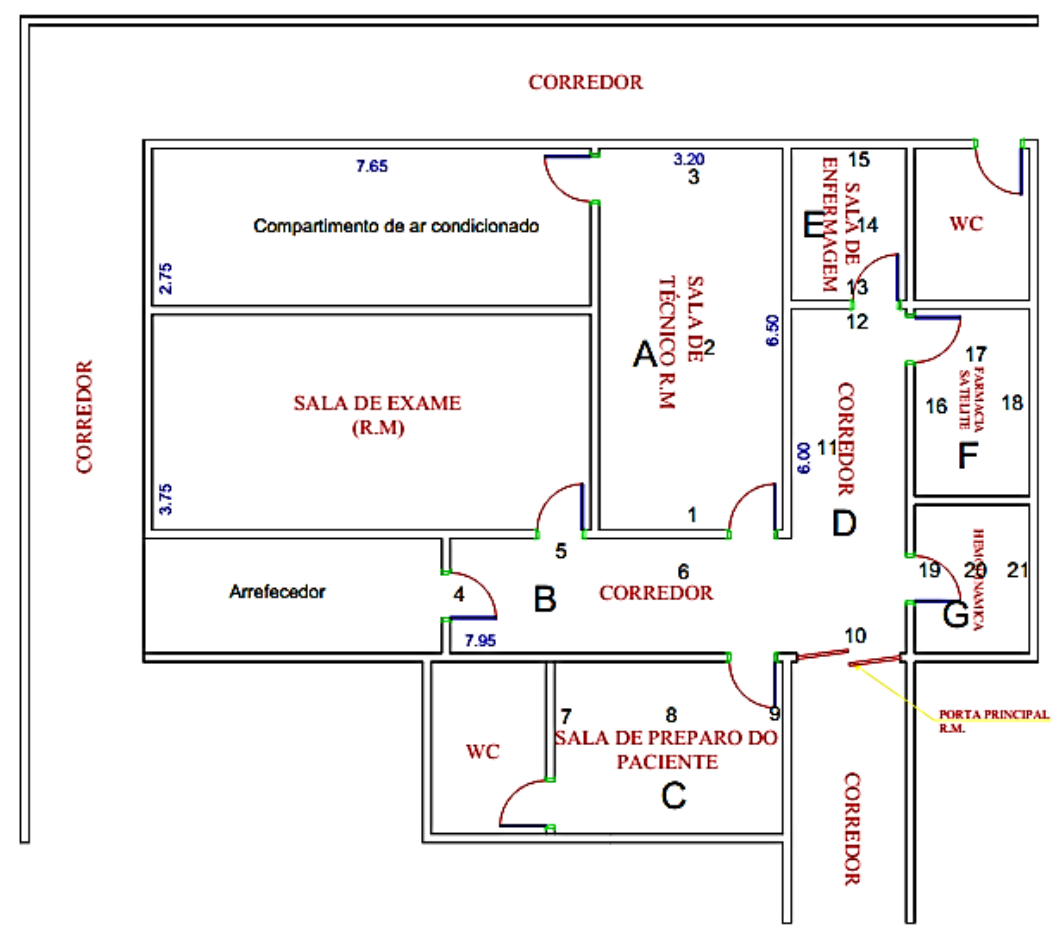

Fonte: Os Autores. 
A Figura 01 indica a classificação dos ambientes estudados neste trabalho, como:

A - Sala do técnico de ressonância magnética (ante - sala).

B - Corredor entre a sala do aparelho e sala de medicação.

C - Sala de Medicação (onde aplica-se o contraste/sala de espera).

D - Corredor entre sala do técnico, farmácia/hemocentro.

E-Sala de Enfermagem.

F - Sala de Farmácia.

E - Sala de Hemocentro.

\section{RESULTADOS}

Em cada ambiente classificado como A, B, C, D, E, F e G, foram realizadas três leituras anotando-se os valores de máximo e mínimo. Assim, para cada local medido uma média foi alcançada a fim de se obter valores com mais precisão e certeza. Os níveis de pressão sonora sem e com a realização de exame foram obtidos e os resultados estão dispostos na Tabela 01.

Tabela 1. Medidas dos Ruídos com ou sem a realização de exame.

\begin{tabular}{|c|c|c|c|c|}
\hline \multirow{2}{*}{$\begin{array}{l}\text { Posições } \\
\text { medidas }\end{array}$} & \multicolumn{2}{|c|}{ Mínimo dB(A) } & \multicolumn{2}{|c|}{ Máximo dB(A) } \\
\hline & sem exame & com exame & sem exame & com exame \\
\hline \multicolumn{5}{|c|}{ A - Sala do técnico de Ressonância Magnética } \\
\hline 1 & 53,8 & 57,2 & 54,7 & 62,5 \\
\hline 2 & 54 & 59,1 & 56,9 & 66,7 \\
\hline 3 & 53,6 & 56,8 & 54,3 & 60,1 \\
\hline Média & 53,8 & 57,7 & 55,3 & 63,1 \\
\hline \multicolumn{5}{|c|}{ B - Corredor entre a sala do aparelho e sala de medicação } \\
\hline 4 & 70,4 & 73,1 & 71,5 & 79,9 \\
\hline 5 & 66 & 67,8 & 68,4 & 72,2 \\
\hline 6 & 64,2 & 66,3 & 67,1 & 69,1 \\
\hline Média & 66,8 & 69 & 69 & 73,6 \\
\hline \multicolumn{5}{|c|}{ C - Sala de Medicação } \\
\hline 7 & 57,3 & 60,6 & 58 & 73,6 \\
\hline 8 & 58,6 & 61,2 & 60,9 & 68,6 \\
\hline 9 & 61,7 & 62,1 & 63,2 & 74,5 \\
\hline Média & 59,2 & 61,3 & 60,7 & 72,2 \\
\hline \multicolumn{5}{|c|}{ D - Corredor entre sala do técnico, farmácia/hemocentro } \\
\hline 10 & 63,8 & 66 & 64,2 & 69 \\
\hline 11 & 64,4 & 67,6 & 65,4 & 68,3 \\
\hline 12 & 63 & 66,4 & 64,3 & 69,5 \\
\hline Média & 63,7 & 66,6 & 64,6 & 68,9 \\
\hline \multicolumn{5}{|c|}{ E - Sala de Enfermagem } \\
\hline 13 & 56,3 & 57,3 & 58,6 & 62,1 \\
\hline
\end{tabular}


Fonte: Os autores

\begin{tabular}{c|c|c|c|c}
14 & 55,6 & 55,9 & 56,7 & 57,7 \\
\hline 15 & 54,7 & 55 & 55,8 & 55,8 \\
\hline Média & $\mathbf{5 5 , 5}$ & $\mathbf{5 6}$ & $\mathbf{5 7}$ & $\mathbf{5 8 , 5}$ \\
\hline \multicolumn{5}{c}{ F - Sala de Farmácia } \\
\hline 16 & 59,6 & 58,3 & 61,2 & 63,6 \\
\hline 17 & 56,6 & 55,9 & 57 & 57,8 \\
\hline 18 & 55 & 55,7 & 56 & 57,6 \\
\hline Média & $\mathbf{5 7}$ & $\mathbf{5 6 , 6}$ & $\mathbf{5 8}$ & $\mathbf{5 9 , 6}$ \\
\hline \multicolumn{5}{|c}{$\mathbf{G}-$ Sala de Hemocentro } \\
\hline 19 & 56,3 & 56,9 & 58,9 & 60,4 \\
\hline 20 & 56 & 56,7 & 57,5 & 59,8 \\
\hline 21 & 55,8 & 56,3 & 57,3 & 58,4 \\
\hline Média & $\mathbf{5 6}$ & $\mathbf{5 6 , 6}$ & $\mathbf{5 7 , 9}$ & $\mathbf{5 9 , 5}$ \\
\hline
\end{tabular}

\section{DISCUSSÃO}

Para fiscalização em âmbito nacional, limites de ruído, vibrações e exposições à produtos químicos para ambientes ocupacionais a NR-15 que regulamenta as "Atividades e Operações Insalubres" e dispõe os limites de tolerância para ruído contínuo ou intermitente os valores de 85 $\mathrm{dB}(\mathrm{A})$ para 8 horas trabalhadas e $90 \mathrm{~dB}(\mathrm{~A})$ para 4 Horas trabalhadas diariamante.

A NHO-01 da FUNDACENTRO, dispõe o tempo máximo diário de exposição permissível em função do nível de ruído os valores de $85 \mathrm{~dB}(\mathrm{~A})$ para 8 horas e $88 \mathrm{~dB}(\mathrm{~A})$ para 4 horas trabalhadas. Segundo a FUNDACENTRO, esta norma baseia-se conforme padrões técnico-modernos internacionais atuais, não havendo compromisso de equivalência com o critério legal, portanto os resultados e interpretação quanto à sua aplicação podem ser diferentes dos parâmetros dispostos pela NR-15.

Na Tabela 02 estão representados os valores médios obtidos em cada ambiente durante o exame, sem exame e o aumento do ruído em porcentagem durante o exame. 
Tabela 2. Valores médios totais.

\begin{tabular}{c|c|c|c}
\hline Locais & $\begin{array}{c}\text { sem } \\
\text { exame } \\
\mathbf{d B}(\mathbf{A})\end{array}$ & $\begin{array}{c}\text { com exame } \\
\mathbf{d B}(\mathbf{A})\end{array}$ & $\begin{array}{c}\text { Aumento do } \\
\text { ruído }\end{array}$ \\
\hline $\mathrm{A}$ & 54,5 & 60,4 & $09,76 \%$ \\
\hline $\mathrm{B}$ & 67,9 & 71,3 & $04,76 \%$ \\
\hline $\mathrm{C}$ & 59,9 & 66,7 & $10,19 \%$ \\
\hline $\mathrm{D}$ & 64,1 & 67,7 & $05,32 \%$ \\
\hline $\mathrm{E}$ & 56,2 & 57,2 & $01,74 \%$ \\
\hline $\mathrm{F}$ & 57,5 & 58,1 & $01,03 \%$ \\
\hline $\mathrm{G}$ & 56,9 & 58,0 & $01,89 \%$ \\
\hline
\end{tabular}

Fonte: Os Autores.

O gráfico a seguir ilustra as variações de nível de pressão sonora nos momentos com e sem exames para cada ambiente estudado.

Grafico 1. Variações de NPS sem e com a realização de Exames.

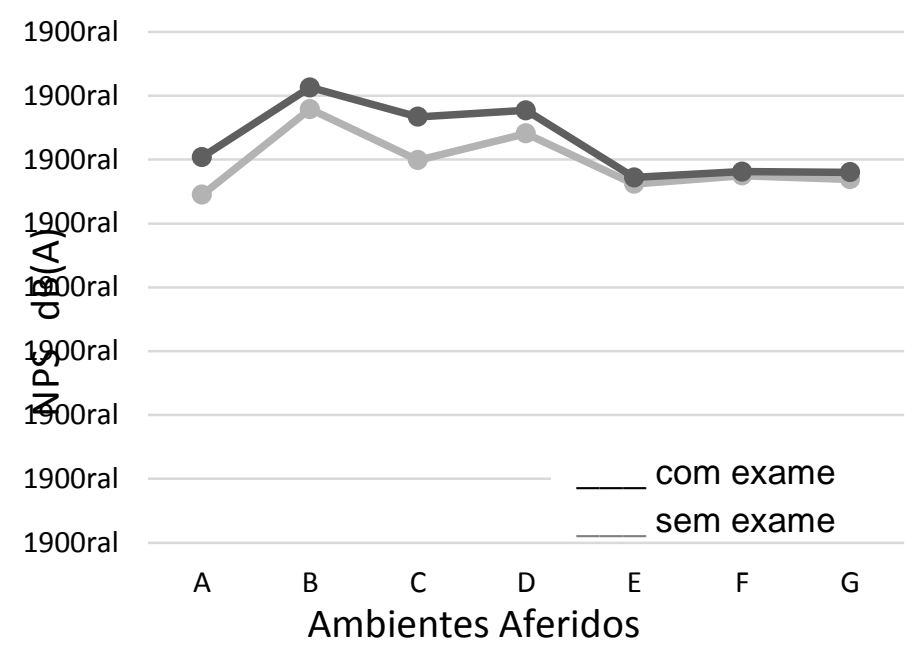

Da Tabela 02 e do Gráfico 01 observa-se que durante o exame houve um aumento significativo de ruído em alguns locais do setor, mas que nenhum apresentou valores acima de 85 $d B(A)$ o estabelecido pela NR-15 e NHO-01.

Verificou-se aumento significativo do nível de pressão sonora durante o exame na sala de medicação e na sala do técnico de ressonância magnética, aumento em 10,19\% e 9,76\%, respectivamente. Nestes dois ambientes, o aumento pode ser explicado pelo fato estarem próximos ao local do aparelho e da sala de arrefecimento.

O corredor, situado entre a sala do aparelho da ressonância magnética, arrefecedor e sala de medicação, apresentou aumento médio de 4,76\%; já o outro corredor, localizado entre a sala do técnico, farmácia e hemocentro, apresentou aumento médio de 5,32\%. 
A farmácia do setor, sala de enfermagem e hemocentro apresentaram aumentos em $1,74 \%, 1,03 \%$ e $1,89 \%$, respectivamente, não sendo portanto, significativos quando comparados aos níveis de ruído sem a realização de exames de ressonância magnética.

\section{CONCLUSÃO}

O presente estudo teve o intuito de colaborar na obtenção de dados sobre o ruído da ressonância magnética e estudar a situação quanto ao nível da pressão sonora em que os funcionários de um Hospital público estão submetidos devido o funcionamento da Ressonância Magnética. Para tanto, foram aferidos os níveis de pressão sonora antes e durante a realização de exames nos ambientes próximos à sala de ressonância magnética.

Em termos de proteção auditiva, vale ressaltar que a jornada de trabalho do setor de ressonância magnética de um Hospital público em Presidente Prudente - SP é de doze horas. Entretanto, há alternância dos funcionários a cada intervalo de quatro horas, seguindo os seguintes horários: das 7:00 hs às $11: 00$ hs, das $11: 00$ às $15: 00$ hs e das $15: 00$ hs às 19:00hs; as oito horas restantes são devidamente cumpridas pelos mesmos em outros setores. Desta forma, esta ação contribui para a proteção auditiva dos funcionários durante o exercício de sua função.

Embora também haja aumento significativo do ruído entre os períodos de realização do exame e o período sem realização dos exames, os valores obtidos se mantiveram abaixo ou próximos aos permissíveis pela legislação, no que diz respeito à proteção auditiva do funcionário durante sua jornada de trabalho no setor.

Ao se comparar os resultados com e sem exames observa-se aumento de ruído em todos os ambientes, mas tanto segundo a NR-15 quanto a NHO-01 os valores máximos ficaram aquém dos limites estabelecidos como limites de tolerâncias para o exercício da profissão quer seja dos enfermeiros, técnicos ou tencólogos.

Este trabalho permite concluir que do ponto de vista da exposição aos ruídos, em ambientes de trabalho, no setor de ressonância magnética de um Hospital público em Presidente Prudente, o nível de pressão sonora não ultrapassa os limites de $85 \mathrm{~dB}(\mathrm{~A})$ recomendados pela NR -15, Anexo no 1 e nem os limites da NHO-01 da FUNDACENTRO.

\section{REFERÊNCIAS}

1. AMARO JÚNIOR, E.; YAMASHITA, H. Aspectos básicos de Tomografia Computadorizada e Ressonância magnética. Revista Brasileira de Psiquiatria, São Paulo, v. 23, suppl. 1, maio 2001. Disponível em: http://dx.doi.org/10.1590/D1516-44462001000500002.. Acesso em: 28 jun. 2012. 
2. ANVISA - Portaria 453/98 - Diretrizes básicas de proteção radiológica em radiodiagnóstico médico e odontológico. DOU 01/06/1998.

3. ASSOCIAÇÃO BRASILEIRA DE NORMAS TÉCNICAS. Referências Bibliográficas: NBR 10151. Rio de Janeiro, 2000; NBR 10152. Rio de Janeiro, 1987.

4. BONTRAGER, K. L.; LAMPIGNANO, J. P. Tratado de Posicionamento Radiográfico e Anatomia Associada. 7. Ed. Rio de Janeiro: Elsevier, 2010.

5. CALIXTO, W. P.; RODRIGUES, C. G. Poluição Sonora. 2004. 103 f. Universidade Católica de Goiás, Goiânia.

6. FANTON, R. Ressonância magnética - Princípio Físico e Aplicação. ed. São Paulo: Escolar, 2007.

7. FUNDACENTRO. Norma de Higiene Ocupacional: NHO - 01. São Paulo, 1999.

8. HOLSBACH, L. R.; CONTO, J. S.; GODOY P. C. C. Avaliação dos níveis de ruído ocupacional em unidades de tratamento intensivo. Sociedad Cubana de Bioingenieria, articulo 00208, mayo. 2001, Habana, Cuba.

9. LEÃO, R. N.; DIAS F. A. M. Perfil audiométrico de indivíduos expostos ao ruído atendidos no núcleo de saúde ocupacional de um hospital do município de Montes Claros, Minas Gerais. Revista CEFAC, São Paulo, v. 12, n. 2, mar./abril. 2010. Disponível em: http://www.scielo.br/scielo.php?script=sci arttext\&pid=s1516-18462010000200010. Acesso em 09 mar. 2012.

10. LI, M.; LIM, T.C.; LEE, J.H. Estudo de simulação em controle ativo de ruído para um 4 Tesla MRI scanner. NIH Public Access, U. S. Department of Health \& Human Services, 01 abril 2009. Disponível em: http://www.ncbi.nlm.nih.gov/pmc/article/PMC2323591/. Acesso em: 12 maio 2012.

11. LOPES, A. C. et al. Condições de saúde auditiva no trabalho: Investigação dos efeitos auditivos em trabalhadores expostos ao ruído ocupacional. Internacional archives of Otorhinolaryngology, jan./mar. 2009. Disponível em: http://www.arquivosdeorl.org.br/conteúdo/acervo port.asp?ld = 588. Acesso em: 17 set. 2012.

12. NORMA REGULAMENTADORA. Atividades e Operações Insalubres: NR - 15. São Paulo, 1992.

13. OLIVEIRA, C. R. D.; ARENAS, G. W. N. Exposição ocupacional a poluição sonora em anestesiologia. Revista Brasileira de Anestesiologia, Campinas, v. 62, n. 2, mar/abril, 2012. Disponivel em: http://www.scielo.br/scielo.php?pid=s0034-

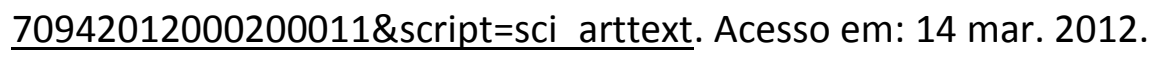

14. OLIVEIRA FILHO, R. H. Uma metodologia para a avaliação virtual da dose de exposição ao ruído no ambiente de trabalho, 2011, 188 f. Tese (Doutorado em engenharia mecânica), programa de pós-graduação em engenharia mecânica- Universidade Federal de Uberlândia. 
15. PORTELA, B. S. Análise da exposição ocupacional ao ruído em motoristas de ônibus urbanos: avaliações objetivas e subjetivas. 2008. 74 f. Dissertação (Mestrado em Engenharia Mecânica) Universidade Federal do Paraná, Curitiba. http://www.pgmec.ufpr.br/dissertaçoes/dissertaçao 103 bruno sergio portela.pdf. Acesso em 04 set. 2012.

16. RAVICZ, M. E.; MELCHER, J. R.; KIANG, N. Y. S. Ruído acústico durante a ressonância magnética funcional. NHI Public Access, U.S. Department of Health \& Human Services, 21 mar. 2008. Disponível em: http://www.ncbi.nlm.nih.gov/pmc/articles/PMC 2270941/. Acesso em: 11 mar. 2012.

17. RODRIGUES, M. N. Metodologia para definição de estratégia de controle e avaliação de ruído ocupacional, 2009, 100f. Dissertação (Mestrado em Engenharia de Estruturas) - Escola de Engenharia Programa de Pós - Graduação em Engenharia de Estruturas, Universidade Federal de Minas Gerais.

18. RUI, L. R.; STEFFANI M. H. Física: Som e Audição Humana, Simpósio Nacional de Ensino de Física, 17. : 2007 jan.29/fev. 02 : São Luís, MA.

19. TINOIS, E. Imagem Funcional - PET E fMRI. MultiCiência Revista Interdisciplinar dos Centros e Núcleos da Unicamp, Ago. 2005.2 Disponível em: http://www.multiciência.unicamp.br/artigos 05/a 02 05.pdf. Acesso em: 28 jun. 2012.

20. WESTBROOK, C.; KAUT, C. Ressonância magnética Aplicada. 2.ed. Rio de Janeiro: Guanabara Koogan, 2000. 